\title{
Long-Term Beneficial Effects of Acupuncture with Reduced Risk of Depression Development Following Trigeminal Neuralgia: A Nationwide Population-Based Cohort Study
}

This article was published in the following Dove Press journal:

Neuropsychiatric Disease and Treatment

\author{
Chung-Chih Liao $\mathbb{D}^{\prime}$ \\ Cheng-Li Lin $\mathbb{1}^{2,3}$ \\ Ke-Ru Liao (iD ${ }^{4}$ \\ Jung-Miao Li (iD) 1,5
}

'Graduate Institute of Chinese Medicine, College of Chinese Medicine, China Medical University, Taichung 40402, Taiwan; ${ }^{2}$ Management Office for Health Data, China Medical University Hospital, Taichung 40447, Taiwan; ${ }^{3}$ College of Medicine, China Medical University, Taichung 40402, Taiwan; ${ }^{4}$ Department of Neurology, Yuanlin Christian Hospital, Yuanlin 51052, Taiwan; ${ }^{5}$ Department of Chinese Medicine, Show Chwan Memorial Hospital, Changhua 50008, Taiwan
Correspondence: Ke-Ru Liao; Jung-Miao Li

Email u940I407@cmu.edu.tw; rungmiau@hotmail.com
Background: Existing evidence has shown that patients with trigeminal neuralgia (TN) have a higher risk of developing depression than the normal population. Clinically, acupuncture has been widely used to alleviate pain in TN. However, few studies have explored the use of acupuncture to prevent depression in TN. Therefore, this study aimed to apply national real-world data to investigate the long-term effect of acupuncture on the risk of depression in patients with TN.

Methods: We recruited participants with newly diagnosed TN from the Taiwanese National Health Insurance Research Database between January 1, 2000 and December 31, 2010, and categorized them into either the acupuncture cohort or non-acupuncture cohort using the 1:1 propensity score-matched method. All patients in the two cohorts were followed up until the end of 2013. Cox proportional hazards regression analysis was used to compare the incidence of depression between the two cohorts.

Results: In total, 776 patients with newly diagnosed TN in each cohort with similar baseline characteristics were enrolled in the study. The acupuncture cohort had a reduced risk of depression compared to the non-acupuncture cohort (adjusted hazard ratio, $0.47 ; 95 \%$ confidence interval, 0.31-0.70). Kaplan-Meier analysis also revealed that the cumulative incidence of depression was significantly lower in the acupuncture cohort during the 13-year follow-up period (Log rank test, $p<0.001$ ). In particular, the beneficial effect of acupuncture was a decrease in the risk of depression among TN patients aged 50-69 years who had also used carbamazepine.

Conclusion: This study demonstrated that acupuncture is associated with a reduction in the risk of depression during long-term follow-up in patients with TN. The results provide new insights for clinical practitioners as well as for health resource allocation.

Keywords: trigeminal neuralgia, depression, real-world data, National Health Insurance Research Database, acupuncture

\section{Introduction}

Trigeminal neuralgia (TN) is a painful neurological disease of the orofacial region. According to the definition of the 3rd edition of the International Classification of Headache Disorders, TN is characterized by recurrent episodes of unilateral, brief electric shock-like pain triggered by innocuous stimuli. The intensity of the pain is severe and is often described as excruciating, which is one of the main reasons related to its burden. The attacks start and end abruptly, and the pain sensation is 
often limited only to the distribution of one or more divisions of the trigeminal nerve. ${ }^{1}$ Pain becomes more prolonged and severe as the TN progresses, and it usually affects the patient's daily activities and quality of life, which may eventually lead to psychosocial distress. ${ }^{2}$

Depression is a common psychiatric disorder, with increasing prevalence worldwide. ${ }^{3}$ Depression usually affects an individual's work performance, causes disability, and is associated with an increased risk of mortality. ${ }^{4-6}$ In particular, concomitant depression with chronic pain due to relapse and TN progression would affect the response and recovery rates of the disease. ${ }^{7,8}$ Moreover, suicide risk may increase, which is a serious life-threatening problem needing much attention., ${ }^{9,10}$ A nationwide retrospective cohort study revealed that depressive disorders were 2.85-fold higher in TN patients than in non-TN patients. ${ }^{11}$ A review study reporting psychometric scores revealed that depression, anxiety, and functional limitation of routine activities could occur in TN patients; therefore, psychological support or treatment is recommended and necessary for TN patients. ${ }^{12}$ In addition, comorbidities with depression in TN patients were associated with multiple invasive surgical procedures; however, limited benefits and high rates of surgical-induced complications were recorded. ${ }^{7}$

Acupuncture has been widely used to treat neuropsychiatric diseases. It has been helpful as an effective treatment for both symptoms of chronic physical pain and depression for thousands of years, globally. ${ }^{13,14}$ A previous case-control study revealed that acupuncture delivers its analgesic effect by decreasing the pain intensity, as evaluated by the Visual Analog Scale in patients with idiopathic TN. ${ }^{15}$ A meta-analysis study revealed that both manual acupuncture and electroacupuncture could significantly improve response and recurrence rates compared to carbamazepine in primary $\mathrm{TN}$ patients. ${ }^{16}$ A clinical study involving 116 patients revealed that acupuncture can improve cognitive function and quality of life in patients with idiopathic TN. ${ }^{17}$ Electroacupuncture and pregabalin could alleviate chronic TN in rats induced by cobra venom; moreover, electroacupuncture could also inhibit the cognitive deficit induced by $\mathrm{TN}^{18}$ However, the effects of acupuncture are usually small, and the quality of the studies is low or very low, mainly due to the lack of adequate controls.

To date, there is still a lack of real-world evidence from a large-scale follow-up to confirm whether acupuncture could reduce the risk of subsequent depression development in patients with TN. Therefore, we designed a nationwide population-based retrospective cohort study to investigate the association between acupuncture treatment and depression development in patients with TN.

\section{Materials and Methods Data Source}

The study was a nationwide, population-based, frequencymatched cohort analysis using the Longitudinal Health Insurance Database 2000 (LHID 2000). The dataset contained all claims data from a random sample of one million beneficiaries from the Taiwanese National Health Insurance Research Database (NHIRD). Data in the LHID 2000 include scrambled, anonymous identification numbers with associated medical records, such as demographic characteristics, clinical visits, prescriptions, procedures, and medical expenditures from 1996 to 2013. The International Classification of Diseases, Ninth Revision, Clinical Modification (ICD-9-CM) was used for the diagnosis codes. The Institutional Review Board of the China Medical University in central Taiwan (CMUH104-REC2 $-115(\mathrm{CR}-4)$ ) approved this study.

\section{Study Population}

The study procedure is summarized in a flowchart shown in Figure 1. We included all subjects newly diagnosed with TN (ICD-9-CM: 350.1), with at least two ambulatory or inpatient claims from January 1, 2000, to December 31, 2010, in the LHID $2000(n=2,303)$. We excluded subjects aged $<18$ years $(n=35)$ and those with a history of depression (ICD-9-CM: 296.2, 296.3, 300.4, 311) before the date of TN diagnosis $(\mathrm{n}=365)$. Finally, 1,903 patients with TN were enrolled between 2000 and 2010 in the LHID 2000. Patients who had received acupuncture treatment after the new date of TN diagnosis were defined as acupuncture users, while the rest were defined as nonacupuncture users. The date of the first acupuncture after the new diagnosis date of TN was defined as the index date for the acupuncture cohort. Regarding the index date for the non-acupuncture users, we randomly assigned a month and day to the same index year of the matched acupuncture cases. The 1:1 propensity score method by sex, age, monthly income, urbanization level, occupation category, baseline comorbidities, and conventional drug use was used to match an equal number of patients in both cohorts. The primary outcome of the study was the incidence of depression, which was diagnosed by qualified 


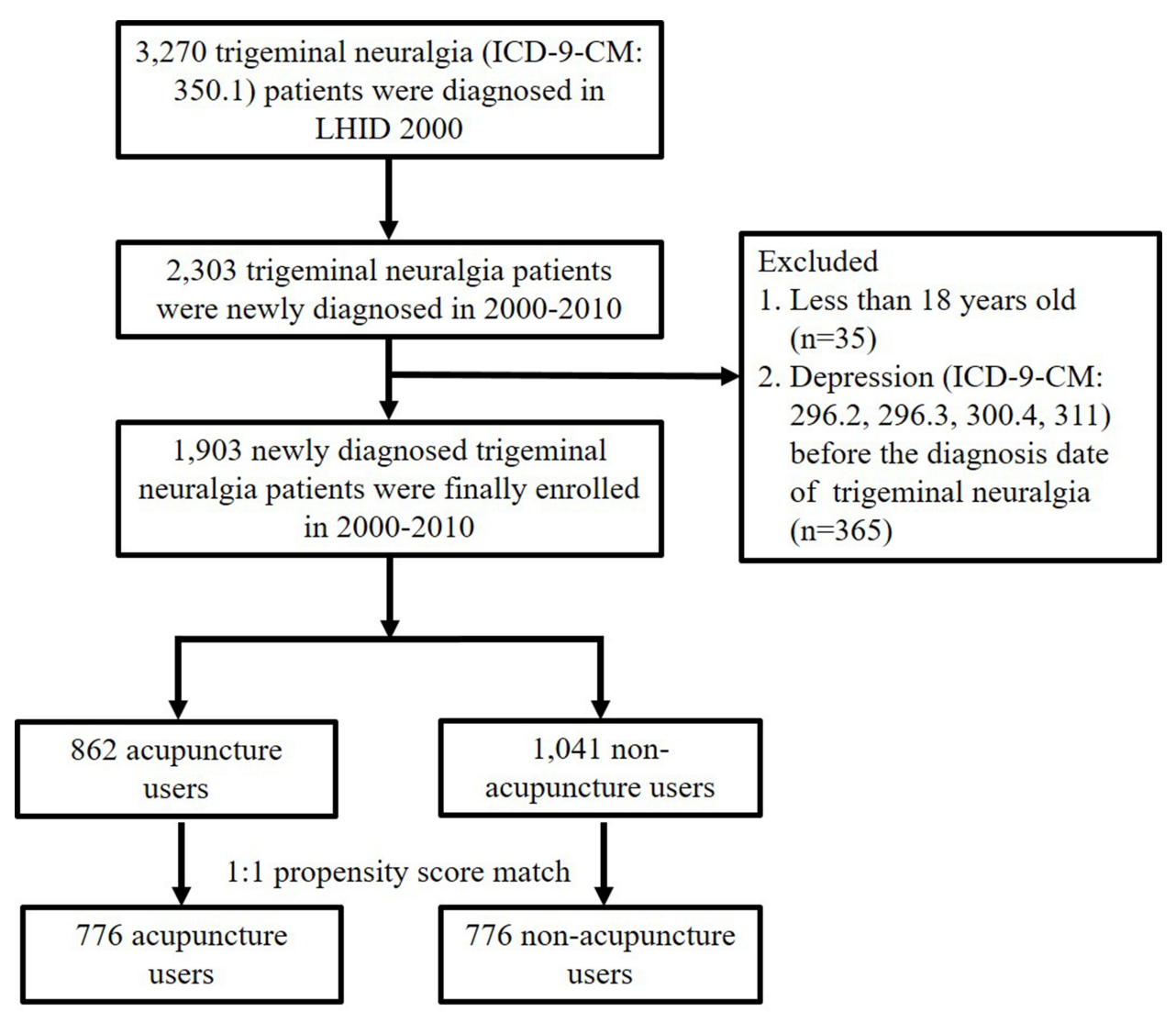

Figure I Flowchart summarizing the study subject enrolment procedure. LHID 2000: Longitudinal Health Insurance Database 2000.

psychiatrists based on medical specialty and ICD-9-CM codes in the database. Therefore, we believe that these data are reliable. The follow-up period started from the index date until diagnosis with a depressive disorder (ICD9-CM: 296.2, 296.3, 300.4, and 311) by a psychiatrist, death, withdrawal from the National Health Insurance (NHI) program, or the end of 2013.

\section{Covariate Assessment}

Covariates considered in our study included sociodemographic factors, baseline comorbidities, and medications. Sociodemographic factors included sex, age, monthly income, urbanization level, and occupation categories. Patients were divided into six subgroups: 18-29, 30-39, $40-49,50-59,60-69$, and $\geq 70$ years. The amount of monthly income was classified into three levels: $<15,000$, 15,000-19,999, and $\geq 20,000$ NT\$/month. Furthermore, we divided the urbanization level of the townships in Taiwan by the population density of the residential area into four levels, with level 1 being the most urbanized and level 4 the least urbanized. The occupation categories included office workers, laborers, and others (including those who were primarily retired, unemployed, and low-income populations). Baseline comorbidities with at least two ambulatory or inpatient claims were considered to exist, including diabetes mellitus (ICD-9-CM 250), hypertension (ICD-9-CM 401-405), hyperlipidemia (ICD-9-CM 272), stroke (ICD-9-CM 430-438), coronary artery disease (ICD-9-CM codes 410-414), mental disorders (ICD-9-CM 290-319, excluding ICD-9-CM: 296.2, 296.3, 300.4, 311), traumatic brain injury (ICD-9-CM 310.2, 800-804, 850-854, 873.x, 910.x, 920.x, 959.1), Parkinson's disease (ICD-9-CM 332), cirrhosis (ICD-9-CM 571), chronic obstructive pulmonary disease (COPD) (ICD-9-CM 491, 492, 496), and renal disease (ICD-9-CM 585). Medications including conventional medicine (carbamazepine, acetaminophen, NSAIDs, morphine, and tramadol) and Chinese herbal medicine (CHM) were also considered in this study.

\section{Statistical Analysis}

Differences in baseline characteristics between both groups were examined using the chi-square test for categorical variables and $t$-test for continuous variables. The incidence rates of depression were calculated for both 
cohorts. Cox proportional hazards regression was performed to evaluate the hazard ratios (HRs) with 95\% confidence intervals (CIs) after adjusting for each covariate. Kaplan-Meier curves and Log rank tests were used to estimate the differences in the cumulative incidence of depression between the two cohorts. A p-value of less than 0.05 indicated statistical significance. All statistical analyses and figures were performed using SAS software, version 9.4 (SAS Institute Inc., Cary, NC, U.S.A.).

\section{Results}

We identified 1,903 patients with newly diagnosed TN who met the inclusion criteria between 2000 and 2010 using the LHID 2000. Among the included subjects, 862 patients $(45.3 \%)$ received acupuncture treatment, while 1,041 patients $(54.7 \%)$ did not receive acupuncture treatment from the initial TN diagnosis date to December 31, 2010. To minimize the interference of confounding variables, we ultimately used a 1:1 propensity score matching method to randomly select 776 patients in the acupuncture and non-acupuncture cohorts. The flow chart is shown in Figure 1. The cumulative dropout rate during 2000-2013 was $8.38 \%$ in the acupuncture cohort, which was slightly lower than that in the non-acupuncture cohort (15.5\%). The possible reasons for the discontinuity of NHI include death, withdrawal of insurance, immigration, prison sentence, etc.

The baseline characteristics of both cohorts are presented in Table 1. After the frequency-matching procedure, there were no significant differences in sex, age, income, urbanization, occupation, baseline comorbidities, and conventional medicine use between the two groups. The proportion ratio of females to males was approximately 6:4. The highest proportion of TN patients in both cohorts were in the 40-49-year-old and 50-59-year-old age groups, and the mean age was approximately 54 years. The most common baseline comorbidity was hypertension (47\%), followed by hyperlipidemia (40\%), and cirrhosis (36\%) in the two groups. All patients received acetaminophen and NSAIDs, and more than $50 \%$ of patients had used carbamazepine for the conventional treatment of TN. After the frequency-matching procedure, we found that $\mathrm{TN}$ patients who received acupuncture treatment were more likely to have received concurrent CHM (97.4\%) compared to non-acupuncture users $(58.4 \%)(\mathrm{p}<0.001)$. The mean duration between the initial diagnosis of TN and the first reception of acupuncture was approximately 941 days. The mean follow-up periods for depression were 5.47 and
Table I Characteristics of Trigeminal Neuralgia Patients According to the Reception of Acupuncture

\begin{tabular}{|c|c|c|c|c|c|}
\hline \multirow[t]{3}{*}{ Variable } & \multicolumn{4}{|c|}{ Received Acupuncture } & \multirow[t]{3}{*}{ p value* } \\
\hline & \multicolumn{2}{|c|}{$\begin{array}{l}\text { No } \\
(n=776)\end{array}$} & \multicolumn{2}{|c|}{$\begin{array}{l}\text { Yes } \\
(n=776)\end{array}$} & \\
\hline & $\mathbf{n}$ & $\%$ & $\mathbf{n}$ & $\%$ & \\
\hline Sex & & & & & 0.72 \\
\hline Female & 475 & 61.2 & 468 & 60.3 & \\
\hline Male & 301 & 38.8 & 308 & 39.7 & \\
\hline Age group & & & & & 0.17 \\
\hline $18-29$ & 51 & 6.57 & 52 & 6.70 & \\
\hline $30-39$ & 104 & 13.4 & 90 & 11.6 & \\
\hline $40-49$ & 179 & 23.1 & 176 & 22.7 & \\
\hline $50-59$ & 162 & 20.9 & 187 & 24.1 & \\
\hline $60-69$ & 132 & 17.0 & 153 & 19.7 & \\
\hline$\geq 70$ & 148 & 19.1 & 118 & 15.2 & \\
\hline Mean \pm SD (years) $^{\dagger}$ & \multicolumn{2}{|c|}{$54.0(15.9)$} & \multicolumn{2}{|c|}{$53.6(14.7)$} & 0.69 \\
\hline Monthly income ${ }^{\star \star \star}$ & & & & & 0.83 \\
\hline$<15,000$ NTD $(<500$ & 226 & 29.1 & 222 & 28.6 & \\
\hline USD) & & & & & \\
\hline 15,000-19,999 NTD & 229 & 29.5 & 240 & 30.9 & \\
\hline $\begin{array}{l}\geq 20,000 \text { NTD }(\geq \\
\quad 260.6\end{array}$ & 321 & 41.4 & 314 & 40.5 & \\
\hline 666.66 USD) & & & & & \\
\hline Urbanization level $^{\&}$ & & & & & 0.96 \\
\hline I (highest) & 255 & 32.9 & 255 & 32.9 & \\
\hline 2 & 216 & 27.8 & 208 & 26.8 & \\
\hline 3 & 112 & 14.4 & 118 & 15.2 & \\
\hline 4 (lowest) & 193 & 24.9 & 195 & 25.1 & \\
\hline \multicolumn{6}{|l|}{ Occupation category ${ }^{\ddagger}$} \\
\hline Office worker & 381 & 49.1 & 408 & 52.6 & \\
\hline Laborer & 328 & 42.3 & 293 & 37.8 & \\
\hline Other & 67 & 8.63 & 75 & 9.66 & \\
\hline \multicolumn{6}{|l|}{ Baseline Comorbidity } \\
\hline Diabetes mellitus & 86 & 11.1 & 90 & 11.6 & 0.75 \\
\hline Hypertension & 365 & 47.0 & 370 & 47.7 & 0.80 \\
\hline Hyperlipidemia & 312 & 40.2 & 309 & 39.8 & 0.88 \\
\hline Stroke & 46 & 5.93 & 44 & 5.67 & 0.83 \\
\hline Coronary artery & 215 & 27.7 & 217 & 28.0 & 0.91 \\
\hline \multicolumn{6}{|l|}{ disease } \\
\hline Mental disorders & 117 & 15.1 & 118 & 15.2 & 0.94 \\
\hline Traumatic brain injury & $14 \mid$ & 18.2 & 137 & 17.7 & 0.79 \\
\hline Parkinson's disease & 14 & 1.80 & 14 & 1.80 & 0.99 \\
\hline Cirrhosis & 283 & 36.5 & 277 & 35.7 & 0.75 \\
\hline COPD & 176 & 22.7 & 180 & 23.2 & 0.81 \\
\hline Renal disease & 39 & 5.03 & 37 & 4.77 & 0.81 \\
\hline \multicolumn{6}{|l|}{$\begin{array}{l}\text { Conventional } \\
\text { medicine }\end{array}$} \\
\hline Carbamazepine & 415 & 53.5 & 448 & 57.7 & 0.09 \\
\hline
\end{tabular}

(Continued) 
Table I (Continued).

\begin{tabular}{|c|c|c|c|c|c|}
\hline \multirow[t]{3}{*}{ Variable } & \multicolumn{4}{|c|}{ Received Acupuncture } & \multirow[t]{3}{*}{ p value* } \\
\hline & \multicolumn{2}{|c|}{$\begin{array}{l}\text { No } \\
(n=776)\end{array}$} & \multicolumn{2}{|c|}{$\begin{array}{l}\text { Yes } \\
(n=776)\end{array}$} & \\
\hline & $\mathbf{n}$ & $\%$ & $\mathbf{n}$ & $\%$ & \\
\hline Acetaminophen & 776 & 100.0 & 776 & 100.0 & - \\
\hline NSAIDs & 776 & 100.0 & 776 & 100.0 & - \\
\hline Morphine & 138 & 17.8 & 110 & 14.2 & 0.05 \\
\hline Tramadol & 223 & 28.7 & 242 & 31.2 & 0.29 \\
\hline $\begin{array}{l}\text { Chinese herbal } \\
\text { medicine }\end{array}$ & 453 & 58.4 & 756 & 97.4 & $<0.001$ \\
\hline $\begin{array}{l}\text { The date of } \\
\text { trigeminal neuralgia } \\
\text { diagnosis (year) }\end{array}$ & & & & & 0.01 \\
\hline 2000 & 89 & 11.5 & 84 & 10.8 & \\
\hline 2001 & 70 & 9.02 & 90 & 11.6 & \\
\hline 2002 & 70 & 9.02 & 65 & 8.38 & \\
\hline 2003 & 66 & 8.51 & 58 & 7.47 & \\
\hline 2004 & 57 & 7.35 & 68 & 8.76 & \\
\hline 2005 & 49 & 6.31 & 82 & 10.6 & \\
\hline 2006 & 67 & 8.63 & 78 & 10.1 & \\
\hline 2007 & 71 & 9.15 & 74 & 9.54 & \\
\hline 2008 & 78 & 10.1 & 64 & 8.25 & \\
\hline 2009 & 70 & 9.02 & 57 & 7.35 & \\
\hline 2010 & 89 & 11.5 & 56 & 7.22 & \\
\hline $\begin{array}{l}\text { Duration between } \\
\text { trigeminal neuralgia } \\
\text { date and index, days } \\
\text { (mean, median) }\end{array}$ & \multicolumn{2}{|c|}{$(1211,944)$} & \multicolumn{2}{|c|}{$(941,617)$} & \\
\hline $\begin{array}{l}\text { Total hospital visits } \\
\text { (mean, median) }\end{array}$ & \multicolumn{2}{|c|}{$(30.3,24)$} & \multicolumn{2}{|c|}{$(37.4,30.4)$} & \\
\hline $\begin{array}{l}\text { Acupuncture visits } \\
\text { (mean, median) }\end{array}$ & & & \multicolumn{2}{|c|}{$(12.1,4)$} & \\
\hline
\end{tabular}

Notes: The mean (median) of the follow-up period for depression was 5.47 (3.52) and 3.50 (3.00) years for the acupuncture cohort and non-acupuncture cohort, respectively; ${ }^{*}$ Chi-square test; ${ }^{\dagger} t$-test; ${ }^{* *}$ NTD, New Taiwan Dollar; I NTD is equal to 0.03 USD; ${ }^{\&}$ The urbanization level was divided by the population density of the residential area into four levels; level I was the most urbanized and level 4 was the least urbanized; ${ }^{\ddagger}$ Other occupation categories included those who were primarily retired, unemployed, and low-income populations.

3.50 years for the acupuncture and non-acupuncture cohorts, respectively. The mean number of acupuncture visits was 12.1 .

Table 2 shows the crude and adjusted HRs (aHR) of depression with covariates among patients with $\mathrm{TN}$ using Cox proportional hazards regression. TN patients receiving acupuncture treatment were less likely to develop depression than those not receiving acupuncture treatment (aHR, 0.47; 95\% CI, 0.31-0.70) after adjusting for sex,
Table 2 Hazard Ratios of Depression with Covariates Among Patients with Trigeminal Neuralgia in Multivariable Cox Proportional Hazards Regression

\begin{tabular}{|c|c|c|}
\hline \multirow[t]{2}{*}{ Variable } & \multicolumn{2}{|l|}{ Depression } \\
\hline & $\begin{array}{l}\text { Crude } \mathbf{H R}^{\dagger} \\
(95 \% \mathrm{Cl})\end{array}$ & $\begin{array}{l}\text { Adjusted } \mathrm{HR}^{\ddagger} \\
(95 \% \mathrm{Cl})\end{array}$ \\
\hline $\begin{array}{l}\text { Received acupuncture } \\
\text { Sex(Male vs Female) }\end{array}$ & $\begin{array}{l}0.48(0.35,0.66) \\
0.75(0.54,1.03)\end{array}$ & $\begin{array}{l}0.47(0.31,0.70)^{* * *} \\
0.82(0.56,1.20)\end{array}$ \\
\hline 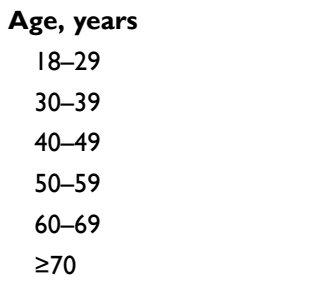 & $\begin{array}{l}1.00 \\
1.45(0.67,3.12) \\
1.12(0.54,2.33) \\
1.16(0.56,2.41) \\
1.37(0.66,2.87) \\
1.79(0.86,3.70)\end{array}$ & $\begin{array}{l}1.00 \\
0.68(0.29,1.58) \\
0.86(0.38,1.94) \\
0.79(0.34,1.86) \\
0.86(0.35,2.12) \\
0.84(0.35,2.01)\end{array}$ \\
\hline $\begin{array}{l}\text { Monthly income }{ }^{* *} \\
<15,000 \text { NTD }(<500 \\
\text { USD) } \\
\quad \text { I5,000-19,999 NTD } \\
(500-666.6 \text { USD) } \\
\geq 20,000 \text { NTD ( } \geq 666.66 \\
\text { USD) }\end{array}$ & $\begin{array}{l}1.00 \\
1.00(0.68,1.45) \\
0.85(0.59,1.21)\end{array}$ & $\begin{array}{l}1.00 \\
2.12(1.22,3.68)^{* *} \\
1.74(1.02,2.97)^{*}\end{array}$ \\
\hline $\begin{array}{l}\text { Urbanization level }^{\&} \\
\quad \text { ( (highest) } \\
2 \\
3 \\
4 \text { (lowest) }\end{array}$ & $\begin{array}{l}1.00 \\
0.80(0.55,1.17) \\
0.71(0.44,1.14) \\
0.82(0.55,1.21)\end{array}$ & $\begin{array}{l}1.00 \\
0.70(0.46,1.07) \\
0.69(0.41,1.18) \\
0.80(0.51,1.23)\end{array}$ \\
\hline $\begin{array}{l}\text { Occupation category } \\
\text { Office worker } \\
\text { Laborer } \\
\text { Other }\end{array}$ & $\begin{array}{l}1.00 \\
1.45(1.05,2.02) \\
2.61(1.69,4.01)^{* * *}\end{array}$ & $\begin{array}{l}1.00 \\
0.75(0.50,1.13) \\
2.37(1.30,4.32)^{* *}\end{array}$ \\
\hline $\begin{array}{l}\text { Baseline comorbidities } \\
\text { (yes vs no) } \\
\text { Diabetes mellitus } \\
\text { Hypertension } \\
\text { Hyperlipidemia } \\
\text { Stroke } \\
\text { Coronary artery disease } \\
\text { Mental disorders } \\
\text { Traumatic brain injury } \\
\text { Parkinson's disease } \\
\text { Cirrhosis } \\
\text { COPD } \\
\text { Renal disease }\end{array}$ & $\begin{array}{l}1.62(1.08,2.43)^{*} \\
1.22(0.91,1.64) \\
1.18(0.88,1.60) \\
1.42(0.79,2.55) \\
1.44(1.05,1.96)^{*} \\
40.7(27.0,61.5)^{* * *} \\
1.03(0.70,1.53) \\
1.50(0.56,4.04) \\
1.22(0.90,1.66) \\
1.24(0.88,1.74) \\
1.14(0.58,2.23)\end{array}$ & $\begin{array}{l}1.32(0.80,2.18) \\
0.75(0.47,1.18) \\
0.92(0.63,1.34) \\
0.89(0.45,1.76) \\
0.89(0.60,1.32) \\
57.9(37.1,90.5) \\
0.90(0.57,1.40) \\
0.71(0.24,2.16) \\
1.09(0.77,1.57) \\
0.78(0.52,1.18) \\
1.11(0.53,2.36)\end{array}$ \\
\hline $\begin{array}{l}\text { Conventional medicine } \\
\text { Carbamazepine } \\
\text { Acetaminophen } \\
\text { NSAIDs } \\
\text { Morphine } \\
\text { Tramadol }\end{array}$ & $\begin{array}{l}0.89(0.58,1.39) \\
0.49(0.33,0.72)^{* * *}\end{array}$ & $\begin{array}{l}1.05(0.65,1.70) \\
0.35(0.23,0.55)^{* * *}\end{array}$ \\
\hline
\end{tabular}

(Continued) 
Table 2 (Continued).

\begin{tabular}{|l|l|l|}
\hline \multirow{2}{*}{ Variable } & \multicolumn{2}{|l|}{ Depression } \\
\cline { 2 - 3 } & $\begin{array}{l}\text { Crude HR } \\
(\mathbf{9 5 \%} \mathbf{~ C l})\end{array}$ & $\begin{array}{l}\text { Adjusted HR } \\
(\mathbf{9 5 \%} \mathbf{~ C l})\end{array}$ \\
\hline $\begin{array}{l}\text { Chinese herbal } \\
\text { medicine }\end{array}$ & $0.37(0.27,0.50)^{* * *}$ & $0.59(0.39,0.90)^{*}$ \\
\hline
\end{tabular}

Notes: ${ }^{\dagger}$ Crude HR, relative hazard ratio; ${ }^{\ddagger}$ Adjusted HR, adjusted for receiving acupuncture, sex, age, monthly income, urbanization level, occupation category, diabetes mellitus, hypertension, hyperlipidemia, stroke, coronary artery disease, mental disorders, traumatic brain injury, Parkinson's disease, cirrhosis, COPD, renal disease, conventional medicine of carbamazepine, morphine, and tramadol, and Chinese herbal medicine in Cox proportional hazards regression; ${ }^{* *} \mathrm{NTD}$, New Taiwan Dollar; I NTD is equal to 0.03 USD; ${ }^{*} p<0.05$, **p $<0.01$; ***p $<0.001$. Abbreviations: $\mathrm{HR}$, hazard ratio; $\mathrm{Cl}$, confidence interval.

age, monthly income, urbanization level, occupation category, baseline comorbidities, and medications used. There was no difference in the risk of depression incidence between males and females (males vs females: aHR, 0.82; 95\% CI, 0.56-1.20). There was also no difference in the risk of depression incidence between any age groups. Among the baseline comorbidities listed, only mental disorders increased the risk of depression (aHR, 57.9; 95\% CI, 37.1-90.5). In addition, participants who took tramadol and CHM had a lower risk of depression (aHR, 0.35; 95\% CI, 0.23.0.55 for tramadol; aHR, 0.59; 95\% CI, 0.39-0.90 for CHM).

Table 3 displays the incidence rates and HRs of depression in acupuncture users compared to non-acupuncture users stratified by sex, age, monthly income, urbanization, occupation categories, comorbidity, and medication use. Among the patients with TN, 69 patients in the acupuncture cohort (16.3 per 1000 person-years) and 104 patients in the non-acupuncture cohort (38.3 per 1000 personyears) developed depression during the follow-up period. In the Cox proportional hazards analysis, the adjusted model showed a significant overall benefit for acupuncture users compared to non-acupuncture users (aHR, 0.47; 95\% CI, 0.31-0.70).

The beneficial effect of acupuncture on the incidence of depression was observed in both female and male patients (aHR, 0.54; 95\% CI, 0.32-0.92 for females; aHR, 0.43 ; 95\% CI, $0.19-0.98$ for males). The benefit of using acupuncture was apparent in patients aged 50-59 years and 60-69 years were less likely to develop depression (aHR, 0.21; 95\% CI, 0.07-0.60; aHR, 0.19; 95\% CI, 0.06-0.64, respectively). Acupuncture decreased the risk of depression in patients with $\mathrm{TN}$ who did not have baseline comorbidities, such as diabetes mellitus, hypertension, hyperlipidemia, stroke, coronary artery disease, traumatic brain injury, Parkinson's disease, and renal disease. Acupuncture use was associated with a significantly lower likelihood of depression, independent of the presence of comorbidities such as mental disorders, cirrhosis, and COPD. Patients who used carbamazepine and CHM had a lower likelihood of developing depression in the acupuncture cohort compared to the non-acupuncture cohort (aHR, 0.47; 95\% CI, 0.30-0.75 for carbamazepine; aHR, $0.52 ; 95 \%$ CI, $0.34-0.78$ for CHM).

We further explored the joint therapeutic effects of acupuncture and carbamazepine on the risk of depression in patients with TN, as shown in Table 4. The patients who used only carbamazepine did not have a lower risk of depression than those who used neither acupuncture nor carbamazepine (aHR, 0.93; 95\% CI, 0.59-1.48; p > 0.05). Patients who used only acupuncture did not also have a lower risk of depression than those who used neither acupuncture nor carbamazepine (aHR, 0.55; 95\% CI, 0.30-1.00; p > 0.05), but the protective effect of acupuncture in reducing depression incidence seems to be better than that of carbamazepine. Moreover, patients who used both acupuncture and carbamazepine had a significantly lower risk of depression than those who did not use acupuncture or carbamazepine (aHR, 0.40; 95\% CI, 0.23-0.71; $\mathrm{p}<0.01$ ).

Figure 2 shows that the cumulative incidence of depression was significantly lower in the acupuncture cohort than in the non-acupuncture cohort during the 13-year follow-up period using the Kaplan-Meier analysis (Log rank test, $\mathrm{p}<0.001$ ).

\section{Discussion}

To the best of our knowledge, this is the first study to use nationwide, large-scale real-world data for long-term follow-up to determine whether acupuncture treatment for TN patients could significantly reduce the risk of depression.

The Taiwanese government established the NHI program in 1995, which covered all public insurance systems and more than $99 \%$ of the 23 million residents now. ${ }^{19,20}$ The NHI program offers health services, including conventional Western medicine and traditional Chinese medicine (TCM). The original claim data of acupuncture and conventional Western medicine utilization were collected from the Taiwanese NHIRD. The NHIRD offered a large population sample to eliminate the bias associated with a limited sample size. In addition, the database provides a time window of over 10 years in terms of the long-term follow-up of 
Table 3 Incidence Rates and Hazard Ratio of Depression for Trigeminal Neuralgia Patients Who Did and Did Not Receive Acupuncture Treatment

\begin{tabular}{|c|c|c|c|c|c|c|c|c|}
\hline \multirow[t]{4}{*}{ Variables } & \multicolumn{6}{|c|}{ Received Acupuncture } & \multicolumn{2}{|c|}{$\begin{array}{l}\text { Compared with Non-Acupuncture } \\
\text { Users }\end{array}$} \\
\hline & \multicolumn{3}{|l|}{ No } & \multicolumn{3}{|l|}{ Yes } & \multirow{3}{*}{$\begin{array}{l}\text { Crude HR } \\
(95 \% \mathrm{Cl})\end{array}$} & \multirow{3}{*}{$\begin{array}{l}\text { Adjusted } \mathbf{H R}^{\dagger} \\
(95 \% \mathrm{Cl})\end{array}$} \\
\hline & \multicolumn{3}{|c|}{$(n=776)$} & \multicolumn{3}{|c|}{$(n=776)$} & & \\
\hline & Event & $\begin{array}{l}\text { Person } \\
\text { Years }\end{array}$ & IR & Event & $\begin{array}{l}\text { Person } \\
\text { Years }\end{array}$ & IR & & \\
\hline Received acupuncture & 104 & 2716 & 38.3 & 69 & 4245 & 16.3 & $0.48(0.35,0.66)^{* * *}$ & $0.47(0.3 \mathrm{I}, 0.70)^{* * *}$ \\
\hline \multicolumn{9}{|l|}{ Sex } \\
\hline Female & 67 & 1706 & 39.3 & 51 & 2620 & 19.5 & $0.57(0.39,0.82)^{* *}$ & $0.54(0.32,0.92)^{*}$ \\
\hline Male & 37 & 1010 & 36.6 & 18 & 1625 & II.I & $0.34(0.19,0.60)^{* * *}$ & $0.43(0.19,0.98)^{*}$ \\
\hline \multicolumn{9}{|l|}{ Age group } \\
\hline $18-29$ & 9 & 173 & 52.1 & 0 & 320 & 0.00 & & \\
\hline $30-39$ & 18 & 391 & 46.0 & 6 & 482 & 12.5 & $0.30(0.12,0.77)^{*}$ & $0.80(0.20,3.28)$ \\
\hline $40-49$ & 20 & 682 & 29.3 & 15 & 970 & 15.5 & $0.59(0.30,1.17)$ & $0.80(0.22,2.90)$ \\
\hline $50-59$ & 19 & 583 & 32.6 & 16 & 1039 & 15.4 & $0.54(0.28,1.06)$ & $0.21(0.07,0.60)^{* *}$ \\
\hline $60-69$ & 20 & 423 & 47.2 & 13 & 849 & 15.3 & $0.36(0.18,0.74)^{* *}$ & $0.19(0.06,0.64)^{* *}$ \\
\hline$\geq 70$ & 18 & 463 & 38.9 & 19 & 585 & 32.5 & $0.88(0.46, \mid .7 I)$ & $0.38(0.13,1.10)$ \\
\hline \multicolumn{9}{|l|}{ Monthly income $e^{* *}$} \\
\hline$<15,000$ NTD $(<500$ USD $)$ & 26 & 849 & 30.6 & 28 & 1205 & 23.2 & $0.81(0.47,1.40)$ & $0.28(0.13,0.63)^{* *}$ \\
\hline $15,000-19,999$ NTD (500-666.6 & 33 & 763 & 43.2 & 22 & 1304 & 16.9 & $0.46(0.26,0.79)^{* *}$ & $0.56(0.27,1.17)$ \\
\hline \multicolumn{9}{|l|}{ USD) } \\
\hline$\geq 20,000$ NTD ( $\geq 666.66$ USD) & 45 & 1104 & 40.8 & 19 & 1736 & 10.9 & $0.3 \mathrm{I}(0.18,0.53)^{* * * *}$ & $0.5 \mathrm{I}(0.24,1.09)$ \\
\hline \multicolumn{9}{|l|}{ Urbanization level $^{\&}$} \\
\hline I (highest) & 35 & 863 & 40.6 & 30 & 1332 & 22.5 & $0.65(0.40,1.06)$ & $0.69(0.34,1.39)$ \\
\hline 2 & 29 & 748 & 38.8 & 15 & 1140 & 13.2 & $0.39(0.21,0.74)$ & $0.46(0.19,1.11)$ \\
\hline 3 & 14 & 433 & 32.3 & 9 & 727 & 12.4 & $0.43(0.19,1.01)$ & $0.21(0.05,0.85)^{*}$ \\
\hline 4 (lowest) & 26 & 673 & 38.7 & 15 & 1046 & 14.3 & $0.4 \mathrm{I}(0.2 \mathrm{I}, 0.77)^{* *}$ & $0.30(0.11,0.78)^{*}$ \\
\hline \multicolumn{9}{|l|}{ Occupation category ${ }^{\ddagger}$} \\
\hline Office worker & 38 & 1383 & 27.5 & 30 & 2242 & 13.4 & $0.56(0.34,0.90)^{*}$ & $0.53(0.28,1.01)$ \\
\hline Laborer & 51 & 1137 & 44.8 & 24 & 1585 & 15.1 & $0.39(0.24,0.63)^{* * *}$ & $0.56(0.29,1.08)$ \\
\hline Other & 15 & 195 & 76.8 & 15 & 418 & 35.9 & $0.54(0.26,1.12)$ & $0.06(0.01,0.29)^{* * * *}$ \\
\hline \multicolumn{9}{|l|}{ Baseline Comorbidity } \\
\hline \multicolumn{9}{|l|}{ Diabetes mellitus } \\
\hline No & 91 & 2465 & 36.9 & 54 & 3785 & 14.3 & $0.44(0.3 \mathrm{I}, 0.6 \mathrm{I})^{* * * *}$ & $0.48(0.31,0.73)^{* * *}$ \\
\hline Yes & 13 & 251 & 51.8 & 15 & 460 & 32.6 & $0.72(0.34,1.56)$ & $0.61(0.14,2.69)$ \\
\hline \multicolumn{9}{|l|}{ Hypertension } \\
\hline No & 55 & 1529 & 36.0 & 30 & 2310 & 13.0 & $0.40(0.26,0.63)^{* * *}$ & $0.42(0.24,0.75)^{* *}$ \\
\hline Yes & 49 & 1187 & 41.3 & 39 & 1935 & 20.2 & $0.56(0.37,0.86)^{* *}$ & $0.63(0.35,1.13)$ \\
\hline \multicolumn{9}{|l|}{ Hyperlipidemia } \\
\hline No & 64 & 1723 & 37.2 & 36 & 2651 & 13.6 & $0.42(0.28,0.63)^{* * *}$ & $0.46(0.27,0.76)^{* *}$ \\
\hline Yes & 40 & 993 & 40.3 & 33 & 1594 & 20.7 & $0.58(0.36,0.93)^{*}$ & $0.74(0.37,1.47)$ \\
\hline \multicolumn{9}{|l|}{ Stroke } \\
\hline No & 99 & 2590 & 38.2 & 62 & 4049 & 15.3 & $0.46(0.33,0.63)^{* * *}$ & $0.56(0.37,0.83)^{* *}$ \\
\hline Yes & 5 & 126 & 39.7 & 7 & 196 & 35.7 & $0.86(0.25,2.92)$ & $0.49(0.00,78.5)$ \\
\hline \multicolumn{9}{|l|}{ Coronary artery disease } \\
\hline No & 74 & 1976 & 37.4 & 38 & 3106 & 12.2 & $0.37(0.25,0.55)^{* * *}$ & $0.44(0.27,0.72)^{* *}$ \\
\hline Yes & 30 & 740 & 40.6 & 31 & 1139 & 27.2 & $0.74(0.45,1.23)$ & $0.88(0.42,1.84)$ \\
\hline
\end{tabular}

(Continued) 
Table 3 (Continued).

\begin{tabular}{|c|c|c|c|c|c|c|c|c|}
\hline \multirow[t]{4}{*}{ Variables } & \multicolumn{6}{|c|}{ Received Acupuncture } & \multicolumn{2}{|c|}{$\begin{array}{l}\text { Compared with Non-Acupuncture } \\
\text { Users }\end{array}$} \\
\hline & \multicolumn{3}{|l|}{ No } & \multicolumn{3}{|l|}{ Yes } & \multirow{3}{*}{$\begin{array}{l}\text { Crude HR } \\
(95 \% \mathrm{Cl})\end{array}$} & \multirow[t]{2}{*}{ Adjusted $\mathbf{H R}^{\dagger}$} \\
\hline & \multicolumn{3}{|c|}{$(n=776)$} & \multicolumn{3}{|c|}{$(n=776)$} & & \\
\hline & Event & $\begin{array}{l}\text { Person } \\
\text { Years }\end{array}$ & IR & Event & $\begin{array}{l}\text { Person } \\
\text { Years }\end{array}$ & IR & & $(95 \% \mathrm{Cl})$ \\
\hline \multicolumn{9}{|c|}{ Mental disorders } \\
\hline No & 20 & 2466 & 8.11 & 7 & 3743 & 1.87 & $0.23(0.10,0.56)^{* *}$ & $0.28(0.10,0.74)^{*}$ \\
\hline Yes & 84 & 250 & 335.8 & 62 & 502 & 123.4 & $0.42(0.30,0.58)^{* * *}$ & $0.62(0.40,0.95)^{*}$ \\
\hline \multicolumn{9}{|c|}{ Traumatic brain injury } \\
\hline No & 87 & 2251 & 38.6 & 56 & 3581 & 15.6 & $0.47(0.33,0.66)^{* * *}$ & $0.56(0.36,0.87)^{* *}$ \\
\hline Yes & 17 & 465 & 36.6 & 13 & 664 & 19.6 & $0.53(0.25,1.11)$ & $0.72(0.24,2.19)$ \\
\hline \multicolumn{9}{|c|}{ Parkinson's disease } \\
\hline No & 101 & 2683 & 37.7 & 68 & 4175 & 16.3 & $0.49(0.36,0.67)^{* * *}$ & $0.53(0.36,0.79)^{* *}$ \\
\hline Yes & 3 & 33 & 89.6 & I & 70 & 14.3 & $0.2 I(0.02,2.1 \mathrm{I})$ & - \\
\hline \multicolumn{9}{|c|}{ Cirrhosis } \\
\hline No & 65 & 1785 & 36.4 & 39 & 2754 & 14.2 & $0.44(0.30,0.66)^{* * *}$ & $0.48(0.28,0.83)^{* *}$ \\
\hline Yes & 39 & 931 & 41.9 & 30 & $149 \mid$ & 20.1 & $0.54(0.33,0.87)^{*}$ & $0.54(0.29,0.99)^{*}$ \\
\hline \multicolumn{9}{|l|}{ COPD } \\
\hline No & 79 & 2154 & 36.7 & 49 & 3286 & 14.9 & $0.47(0.33,00.67)^{* * *}$ & $0.55(0.35,0.87)^{*}$ \\
\hline Yes & 25 & 562 & 44.5 & 20 & 959 & 20.9 & $0.50(0.27,0.91)^{*}$ & $0.38(0.17,0.84)^{*}$ \\
\hline \multicolumn{9}{|c|}{ Renal disease } \\
\hline No & 101 & 2601 & 38.8 & 63 & 4061 & 15.5 & $0.46(0.33,0.63)^{* * *}$ & $0.53(0.36,0.79)^{* *}$ \\
\hline Yes & 3 & 115 & 26.1 & 6 & 184 & 32.7 & $0.98(0.22,4.34)$ & - \\
\hline \multicolumn{9}{|c|}{ Conventional medicine } \\
\hline \multicolumn{9}{|c|}{ Carbamazepine } \\
\hline No & 33 & 1242 & 26.6 & 23 & 1773 & 13.0 & $0.59(0.34,1.01)$ & $0.74(0.34, \mid \quad 0.61)$ \\
\hline Yes & 71 & 1474 & 48.2 & 46 & 2472 & 18.6 & $0.42(0.29,00.61)^{* * *}$ & $0.47(0.30,00.75)^{\text {*** }}$ \\
\hline \multicolumn{9}{|c|}{ Morphine } \\
\hline No & 93 & 2288 & 40.6 & 57 & 3702 & 15.4 & $0.43(0.31,00.61)^{* * *}$ & $0.49(0.32,0.75)^{* * *}$ \\
\hline Yes & 11 & 428 & 25.7 & 12 & 543 & 22.1 & $0.91(0.40,2.08)$ & $3.32(0.49,22.5)$ \\
\hline \multicolumn{9}{|c|}{ Tramadol } \\
\hline No & 90 & 1961 & 45.9 & 53 & 2930 & 18.1 & $0.47(0.33,0.66)^{* * *}$ & $0.56(0.36,0.85)^{* *}$ \\
\hline Yes & 14 & 755 & 18.5 & 16 & 1315 & 12.2 & $0.57(0.27,1.21)$ & $0.48(0.16,1.39)$ \\
\hline \multicolumn{9}{|c|}{ Chinese herbal medicine } \\
\hline No & 60 & 969 & 61.9 & 2 & 100 & 19.9 & $0.43(0.10,1.76)$ & $0.28(0.05,1.69)$ \\
\hline Yes & 44 & 1747 & 25.2 & 67 & 4145 & 16.2 & $0.68(0.46,1.00)$ & $0.52(0.34,0.78)^{* *}$ \\
\hline
\end{tabular}

Notes: ${ }^{\dagger}$ Adjusted HR, adjusted for receiving acupuncture, sex, age, monthly income, urbanization level, occupation category, diabetes mellitus, hypertension, hyperlipidemia, stroke, coronary artery disease, mental disorders, traumatic brain injury, Parkinson's disease, cirrhosis, COPD, renal disease, conventional medicine of carbamazepine, morphine, and tramadol, and Chinese herbal medicine in Cox proportional hazards regression; ${ }^{* *} \mathrm{NTD}$, New Taiwan Dollar; I NTD is equal to 0.03 USD; ${ }^{\&}$ The urbanization level was divided by the population density of the residential area into four levels; level I was the most urbanized and level 4 was the least urbanized; ${ }^{\ddagger}$ Other occupation categories included those who were primarily retired, unemployed, and low-income populations; $*_{p}<0.05$; **p $<0.01$; *** $p<0.001$.

Abbreviations: IR, incidence rates, per I,000 person-years; HR, hazard ratio; $\mathrm{Cl}$, confidence interval.

a cohort, which is convenient, saves time and work effort, and is a tool for observing some chronic disorders like depression in our study, compared to clinical trials. Hence, we considered the database an excellent tool to supply realworld evidence regarding the beneficial effect of applying integrative therapies of acupuncture and conventional
Western medicine on depression development in patients with TN.

The incidence rate of $\mathrm{TN}$ was approximately 12 in 100,000 persons per year, occurring predominantly in the 37-67 years age group, and the proportion of females to males was $3: 1,{ }^{21,22}$ which was similar to the sex and age 
Table 4 Joint Effects of Carbamazepine and Acupuncture on the Risk of Depression in Patients with Trigeminal Neuralgia

\begin{tabular}{|l|l|l|l|l|l|l|}
\hline \multicolumn{2}{|l|}{ Variables } & N & Event & $\begin{array}{l}\text { Crude HR } \\
(95 \% \mathbf{C l})\end{array}$ & $\begin{array}{l}\text { Adjusted HR } \\
\mathbf{( 9 5 \% ~ C l ) ~}\end{array}$ \\
\hline Received acupuncture & Carbamazepine & & & & \\
No & No & $36 I$ & 33 & 1.00 & 1.00 & 0.43 \\
No & Yes & 415 & 71 & $1.85(1.23,2.80)^{* *}$ & $0.93(0.59,1.48)$ \\
Yes & No & 328 & 23 & $0.56(0.33,0.96)^{*}$ & $0.55(0.30,1.00)$ \\
Yes & Yes & 448 & 46 & $0.80(0.5 \mathrm{I}, 1.26)$ & $0.40(0.23,0.71)^{* *}$ \\
\hline
\end{tabular}

Notes: Adjusted HR, adjusted for age, sex, monthly income, urbanization level, occupation category, baseline comorbidities, conventional medicine of carbamazepine, morphine, tramadol, and Chinese herbal medicine; ${ }^{\#} \mathrm{p}$-value for interaction; ${ }^{*} \mathrm{p}<0.05$; ${ }^{* *} \mathrm{p}<0.01$.

Abbreviations: $\mathrm{HR}$, hazard ratio; $\mathrm{Cl}$, confidence interval.

distribution of TN patients reported in our present study that was retrieved from the NHIRD. Chronic pain affects not only physical health but is also associated with psychiatric disorders. ${ }^{23,24}$ Our study found that the beneficial effect of acupuncture in reducing new-onset depression is significant in patients with TN aged between and 50-59 years and 60-69 years. It is well-known that complex mental-physical comorbidities are puzzling problems in middle-aged and older adults than in young adults. This finding suggests that the middle-to-older-aged population has a higher TN susceptibility population and might have a more vigorous attitude than the young regarding receiving combined acupuncture and conventional Western medicine therapy that could substantially reduce depression.

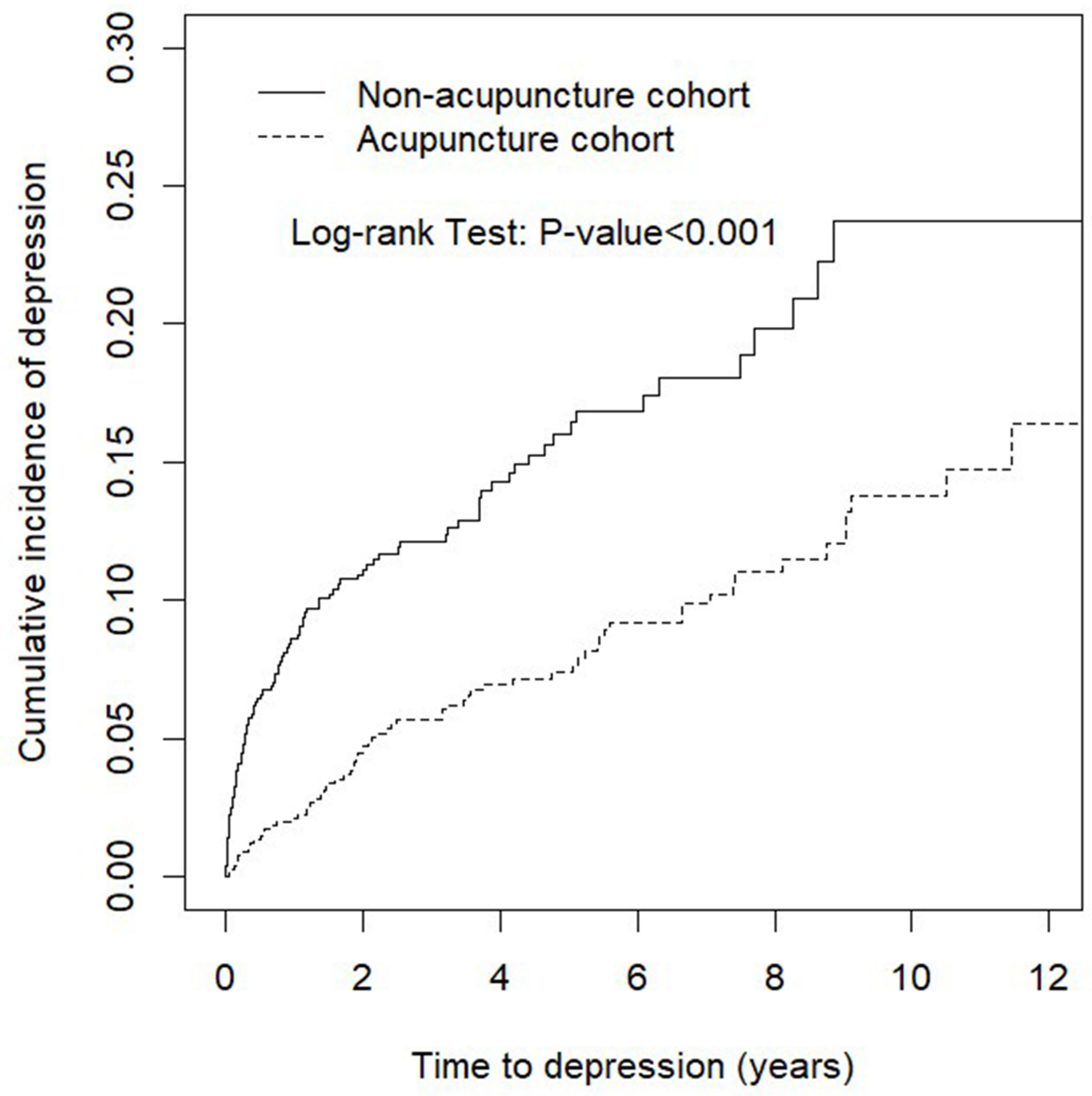

Figure 2 Cumulative incidence of depression between the acupuncture cohort and the non-acupuncture cohort. 
These may have great advantages not only in terms of selfwell-being but also on the family burden and social behavior.

$\mathrm{TN}$ treatment includes pharmacotherapy and surgical intervention. Carbamazepine is a widely accepted first-line pharmacological therapy (200-1200 mg/day), and it is effective (level A) to treat $\mathrm{TN}^{25}$ Some reports also indicated that carbamazepine might be considered as an alternative medication for depressive disorders. ${ }^{26-28}$ Acupuncture is also known to have benefits in depression other than the well-known pain-relief efficacy. ${ }^{29,30}$ A systematic review and meta-analysis also concluded that the therapeutic efficacy of acupuncture combined with antidepressant medication is superior to that of only antidepressant therapy in the short-term period. ${ }^{31}$ Therefore, we are curious to know if carbamazepine or acupuncture use in patients with $\mathrm{TN}$ would reduce depression attacks. However, in the present study, we noticed that the risk of depression in patients with $\mathrm{TN}$ receiving carbamazepine alone was not lower than that of patients who received neither carbamazepine nor acupuncture. Acupuncture alone treatment was observed to reduce depression attacks compared to no carbamazepine/acupuncture treatment before adjusting for covariates (crude $\mathrm{HR}, 0.56$; 95\% CI, $0.33-0.96 ; \mathrm{p}<0.05)$. However, integrative therapy with acupuncture and carbamazepine could substantially reduce the risk of depression in TN patients (aHR, 0.40; 95\% CI, $0.23-0.71 ; \mathrm{p}<0.01)$ compared to neither carbamazepine nor acupuncture. The results suggest that integrative therapy with carbamazepine and acupuncture might be a better option for treating $\mathrm{TN}$ in routine clinical practice and prophylaxis to prevent depression development. In addition, although most patients with TN could get pain relief under conventional medicine use, surgical intervention would be considered for patients who are refractory to conventional pharmacotherapy or have intolerable adverse effects. ${ }^{32}$ Currently, surgical interventions, including microvascular decompression (MVD), stereotactic radiosurgery, and radiofrequency coagulation, have been reported to be effective in treating drug-resistant $\mathrm{TN}^{33,34}$ A report showed that MVD not only increased the rate of pain relief but also substantially improved depression symptoms at the 6-month follow-up. ${ }^{35}$ However, postoperative complications of MVD, such as cerebrospinal fluid leak, wound infection, and hearing loss, are still concerns. ${ }^{36}$ Acupuncture is a minimally invasive procedure with little adverse effects or reported complications compared to current neurosurgery that provides rapid, convenient, and safe pain-relieving effects, independent of age, sex, health status, or race. ${ }^{37,38}$ Our results also proved its long-term beneficial effect on the risk of depression in TN, suggesting that acupuncture should be considered as a complementary and alternative therapy for treating $\mathrm{TN}$ and that patients might consider acupuncture therapy before deciding to receive surgical intervention. Besides, both acupuncture and CHM are key components of TCM in clinical practice in Taiwan. ${ }^{39}$ Our study found that TN patients who received acupuncture treatment simultaneously had very high proportions who also received CHM treatment (97.4\%); CHM also obviously lowered the risk of depression (aHR, 0.59; 95\% CI, 0.39-0.90). However, the role of CHM in treating TN needs to be explored further, especially the prescription patterns of formula and single herb, active ingredients, and how it works.

Newer insights into neural mechanisms in the brain have been rapidly developed to understand the association between chronic pain and depression in recent years. ${ }^{40}$ Acupuncture therapy has also proven beneficial in providing relief to depression associated with chronic pain or distress in experimental research. For example, our earlier study suggested that auricular electroacupuncture could alleviate depression behavior in a nitroglycerin-induced migraine rat model, possibly related to the CGRP/COX2/TRPV1/TRPA1 signaling pathway. ${ }^{41}$ In addition, acupuncture could relieve depression-like behavior in the chronic restraint stress mice model by modulating the central brain neural activity and serotonin receptor. ${ }^{42}$ Acupuncture relief for depressive disorders was proven by regulating the mTOR signal, neuropeptide Y, ERKCREB pathway, glial glutamate transporter, and brainderived neurotrophic factor. ${ }^{42}$ Decreased serotonin and norepinephrine levels in the central nervous system, chronic inflammation, and increase of glutamate are related to the increased risk of developing depression in TN. ${ }^{11}$ The mechanism should be disclosed through experiments in the future to support our clinical data, which showed that acupuncture is beneficial for decreasing the risk of depression in patients with $\mathrm{TN}$.

Our study had some limitations. First, the dataset used in the study could not provide a quantitative approach, such as scale assessment or behavioral parameters, on how acupuncture treatment could alleviate depression in patients with TN. Moreover, it is relatively possible that patients with more uncontrolled $\mathrm{TN}$ or patients with more pain have more psychological suffering than those with lower levels of pain. Therefore, the differences at baseline in this 
outcome could have potentially influenced the reported results. However, information on pain intensity or $\mathrm{TN}$ "severity" could not be obtained from the dataset used in the study. Second, the information related to actual acupuncture points in clinical practice that is effective in reducing depression development could not be acquired from the database. Where acupuncture was performed and how many sessions were conducted individually are related to the effects of acupuncture; however, further analysis of the differences was not mentioned and explored in the present study. In addition, due to the nature of the study and its design, the lack of sham control should be acknowledged in the limitations given that acupuncture has been widely associated with non-specific effects, including placebo. Third, although we included several clinical conditions as covariates, some important diseases, such as sleep disorders or other chronic pain conditions, that are potentially closely associated with depression were not included in the study. Other surgical intervention treatments besides medications, such as MVD or rhizotomy, were also not considered in the present study. These are worthy of further research in the future. Finally, the current study was retrospective in nature. Further prospective clinical trials or animal experiments are needed to further explore the regulatory effects and mechanisms of acupuncture on depression in $\mathrm{TN}$.

\section{Conclusion}

In conclusion, the present study demonstrated that patients with $\mathrm{TN}$ receiving acupuncture treatment could have a reduced risk of subsequent depression development compared to those who did not receive acupuncture treatment. These findings support the use of acupuncture as a complementary therapy for TN, which is worthy of use in clinical practice worldwide.

\section{Abbreviations}

CHM, Chinese herbal medicine; CI, confidence interval; HR, hazard ratio; ICD-9-CM, International Classification of Diseases, Ninth Revision; LHID, Longitudinal Health Insurance Database; NHIRD, National Health Insurance Research Database; TCM, traditional Chinese medicine; $\mathrm{TN}$, trigeminal neuralgia.

\section{Data Sharing Statement}

The data in this study are available to other researchers upon request.

\section{Ethics Approval}

All data accessed from the LHID complies with relevant data protection and privacy regulations.

This study was approved by the Institutional Review Board of China Medical University in central Taiwan (CMUH104-REC2-115(CR-4)).

\section{Acknowledgment}

This study is supported in part by the Taiwan Ministry of Health and Welfare Clinical Trial Center (MOHW109TDU-B-212-114004), MOST Clinical Trial Consortium for Stroke (MOST 108-2321-B-039-003-), Tseng-Lien Lin Foundation, Taichung, Taiwan.

\section{Author Contributions}

All authors made a significant contribution to the work reported, whether that is in the conception, study design, execution, acquisition of data, analysis and interpretation, or in all these areas; took part in drafting, revising or critically reviewing the article; gave final approval of the version to be published; have agreed on the journal to which the article has been submitted; and agree to be accountable for all aspects of the work.

These authors are co-corresponding authors: Ke-Ru Liao and Jung-Miao Li.

\section{Disclosure}

The authors report no conflicts of interest for this work.

\section{References}

1. Arnold M. Headache Classification Committee of the International Headache Society (IHS). The international classification of headache disorders. Cephalalgia. 2018;38(1):1-211. doi:10.1177/03331024177 38202

2. Melek LN, Smith JG, Karamat A, Renton T. Comparison of the neuropathic pain symptoms and psychosocial impacts of trigeminal neuralgia and painful posttraumatic trigeminal neuropathy. J Oral Facial Pain Headache. 2019;33(1):77-88. doi:10.11607/ofph.2157

3. Hidaka BH. Depression as a disease of modernity: explanations for increasing prevalence. $J$ Affect Disord. 2012;140(3):205-214. doi:10.1016/j.jad.2011.12.036

4. Lerner D, Adler DA, Rogers WH, et al. Work performance of employees with depression: the impact of work stressors. Am J Health Promot. 2010;24(3):205-213. doi:10.4278/ajhp.090313-QUAN-103

5. Friedrich MJ. Depression is the leading cause of disability around the world. JAMA. 2017;317(15):1517.

6. Gilman SE, Sucha E, Kingsbury M, Horton NJ, Murphy JM, Colman I. Depression and mortality in a longitudinal study: 1952-2011. Cmaj. 2017;189(42):E1304-e1310. doi:10.1503/cmaj.170125

7. Mousavi SH, Sekula RF, Gildengers A, Gardner P, Lunsford LD. Concomitant depression and anxiety negatively affect pain outcomes in surgically managed young patients with trigeminal neuralgia: long-term clinical outcome. Surg Neurol Int. 2016;7:98. doi:10.4103/ 2152-7806.194145 
8. Rudich Z, Lerman SF, Gurevich B, Shahar G. Pain specialists' evaluation of patient's prognosis during the first visit predicts subsequent depression and the affective dimension of pain. Pain Med. 2010;11(3):446-452. doi:10.1111/j.1526-4637.2009.00795.x

9. Hooley JM, Franklin JC, Nock MK. Chronic pain and suicide: understanding the association. Curr Pain Headache Rep. 2014;18(8):435. doi:10.1007/s11916-014-0435-2

10. Adams H, Pendleton C, Latimer K, Cohen-Gadol AA, Carson BS, Quinones-Hinojosa A. Harvey Cushing's case series of trigeminal neuralgia at the Johns Hopkins Hospital: a surgeon's quest to advance the treatment of the 'suicide disease'. Acta Neurochir (Wien). 2011;153(5):1043-1050. doi:10.1007/s00701-011-0975-8

11. Wu TH, Hu LY, Lu T, et al. Risk of psychiatric disorders following trigeminal neuralgia: a nationwide population-based retrospective cohort study. J Headache Pain. 2015;16:64. doi:10.1186/s10194015-0548-y

12. Melek LN, Devine M, Renton T. The psychosocial impact of orofacial pain in trigeminal neuralgia patients: a systematic review. Int J Oral Maxillofac Surg. 2018;47(7):869-878. doi:10.1016/j. ijom.2018.02.006

13. Vickers AJ, Linde K. Acupuncture for chronic pain. JAMA. 2014;311 (9):955-956. doi:10.1001/jama.2013.285478

14. Smith CA, Armour M, Lee MS, Wang LQ, Hay PJ. Acupuncture for depression. Cochrane Database Syst Rev. 2018;3:Cd004046.

15. Ichida MC, Zemuner M, Hosomi J, et al. Acupuncture treatment for idiopathic trigeminal neuralgia: A longitudinal case-control double blinded study. Chin J Integr Med. 2017;23(11):829-836. doi:10.1007/ s11655-017-2786-0

16. Hu H, Chen L, Ma R, Gao H, Fang J. Acupuncture for primary trigeminal neuralgia: a systematic review and PRISMA-compliant meta-analysis. Complement Ther Clin Pract. 2019;34:254-267. doi:10.1016/j.ctcp.2018.12.013

17. Gao J, Zhao C, Jiang W, Zheng B, He Y. Effect of acupuncture on cognitive function and quality of life in patients with idiopathic trigeminal neuralgia. J Nerv Ment Dis. 2019;207(3):171-174. doi:10.1097/NMD.0000000000000937

18. Chen RW, Liu H, An JX, et al. Cognitive effects of electro-acupuncture and pregabalin in a trigeminal neuralgia rat model induced by cobra venom. J Pain Res. 2017;10:1887-1897. doi: $10.2147 /$ JPR.S140840

19. Yeh JJ, Syue SH, Lin CL, Hsu CY, Shae Z, Kao CH. Effects of statins on anxiety and depression in patients with asthma-chronic obstructive pulmonary disease overlap syndrome. $J$ Affect Disord. 2019;253:277-284. doi:10.1016/j.jad.2019.05.002

20. Wu MY, Lee YC, Lin CL, Huang MC, Sun MF, Yen HR. Trends in use of acupuncture among adults in Taiwan from 2002 to 2011: a nationwide population-based study. PLoS One. 2018;13(4): e0195490. doi:10.1371/journal.pone. 0195490

21. De Toledo IP, Conti Réus J, Fernandes M, et al. Prevalence of trigeminal neuralgia: A systematic review. $J$ Am Dent Assoc. 2016;147(7):570-576.e572. doi:10.1016/j.adaj.2016.02.014

22. Majeed MH, Arooj S, Khokhar MA, Mirza T, Ali AA, Bajwa ZH. Trigeminal neuralgia: a clinical review for the general physician. Cureus. 2018;10(12):e3750.

23. Subramaniam M, Vaingankar JA, Abdin E, Chong SA. Psychiatric morbidity in pain conditions: results from the Singapore Mental Health Study. Pain Res Manag. 2013;18(4):185-190. doi:10.1155/ 2013/798205

24. Barry DT, Cutter CJ, Beitel M, Kerns RD, Liong C, Schottenfeld RS. Psychiatric disorders among patients seeking treatment for co-occurring chronic pain and opioid use disorder. $J$ Clin Psychiatry. 2016;77(10):1413-1419. doi:10.4088/JCP.15m09963

25. Obermann M. Treatment options in trigeminal neuralgia. Ther $A d v$ Neurol Disord. 2010;3(2):107-115. doi:10.1177/1756285609359317
26. Zhang ZJ, Tan QR, Tong Y, et al. The effectiveness of carbamazepine in unipolar depression: a double-blind, randomized, placebo-controlled study. J Affect Disord. 2008;109(1-2):91-97. doi:10.1016/j.jad.2007.11.004

27. Post RM, Uhde TW, Roy-Byrne PP, Joffe RT. Antidepressant effects of carbamazepine. Am J Psychiatry. 1986;143(1):29-34.

28. Vigo DV, Baldessarini RJ. Anticonvulsants in the treatment of major depressive disorder: an overview. Harv Rev Psychiatry. 2009;17 (4):231-241. doi:10.1080/10673220903129814

29. Liao CC, Liao KR, Lin CL, Li JM. Long-term effect of acupuncture on the medical expenditure and risk of depression and anxiety in migraine patients: a retrospective cohort study. Front Neurol. 2020;11:321. doi:10.3389/fneur.2020.00321

30. Yang Z, Zhao L, Xie X, et al. The effectiveness of acupuncture for chronic pain with depression: a systematic review protocol. Medicine (Baltimore). 2017;96(47):e8800. doi:10.1097/MD.0000000000008 800

31. Chan YY, Lo WY, Yang SN, Chen YH, Lin JG. The benefit of combined acupuncture and antidepressant medication for depression: a systematic review and meta-analysis. $J$ Affect Disord. 2015;176:106-117. doi:10.1016/j.jad.2015.01.048

32. Montano N, Conforti G, Di Bonaventura R, Meglio M, Fernandez E, Papacci F. Advances in diagnosis and treatment of trigeminal neuralgia. Ther Clin Risk Manag. 2015;11:289-299. doi:10.2147/ TCRM.S37592

33. Li Y, Yang L, Ni J, Dou Z. Microvascular decompression and radiofrequency for the treatment of trigeminal neuralgia: a meta-analysis. $J$ Pain Res. 2019;12:1937-1945. doi:10.2147/JPR.S203141

34. Tuleasca C, Régis J, Sahgal A, et al. Stereotactic radiosurgery for trigeminal neuralgia: a systematic review. J Neurosurg. 2018;130 (3):733-757.

35. Cheng J, Long J, Hui X, Lei D, Zhang H. Effects of microvascular decompression on depression and anxiety in trigeminal neuralgia: a prospective cohort study focused on risk factors and prognosis. Clin Neurol Neurosurg. 2017;161:59-64. doi:10.1016/j.clineuro. 2017.08.011

36. Hannan C, Shoakazemi A, Quigley G. Microvascular decompression for trigeminal neuralgia: a regional unit's experience. Ulster Med J. 2018;87(1):30-33.

37. Vincent C. The safety of acupuncture. BMJ. 2001;323 (7311):467-468. doi:10.1136/bmj.323.7311.467

38. Xiang A, Cheng K, Shen X, Xu P, Liu S. The immediate analgesic effect of acupuncture for pain: a systematic review and meta-analysis. Evid Based Complement Alternat Med. 2017;2017:3837194. doi:10.1155/2017/3837194

39. Ton G, Liao HY, Chiang JH, Chen YH, Lee YC. Chinese herbal medicine and acupuncture reduced the risk of stroke after Bell's Palsy: a population-based retrospective cohort study. $J$ Altern Complement Med. 2019;25(9):946-956. doi:10.1089/acm.2018.0496

40. Sheng J, Liu S, Wang Y, Cui R, Zhang X. The link between depression and chronic pain: neural mechanisms in the brain. Neural Plast. 2017;2017:9724371. doi:10.1155/2017/9724371

41. Liao CC, Li JM, Hsieh CL. Auricular electrical stimulation alleviates headache through CGRP/COX-2/TRPV1/TRPA1 signaling pathways in a nitroglycerin-induced migraine rat model. Evid Based Complement Alternat Med. 2019;2019:2413919.

42. Lee MJ, Ryu JS, Won SK, et al. Effects of acupuncture on chronic stress-induced depression-like behavior and its central neural mechanism. Front Psychol. 2019;10:1353. doi:10.3389/ fpsyg.2019.01353 


\section{Publish your work in this journal}

Neuropsychiatric Disease and Treatment is an international, peerreviewed journal of clinical therapeutics and pharmacology focusing on concise rapid reporting of clinical or pre-clinical studies on a range of neuropsychiatric and neurological disorders. This journal is indexed on PubMed Central, the 'PsycINFO' database and CAS, and is the official journal of The International Neuropsychiatric Association (INA). The manuscript management system is completely online and includes a very quick and fair peer-review system, which is all easy to use. Visit http://www.dovepress.com/testimonials.php to read real quotes from published authors.

Submit your manuscript here: https://www.dovepress.com/neuropsychiatric-disease-and-treatment-journal 